\title{
Towards Networked Computers: What Can Be Learned from Distributed Computing?
}

\author{
David Peleg \\ Department of Computer Science and Applied Mathematics \\ The Weizmann Institute of Science, Israel
}

\begin{abstract}
The talk will discuss some key ideas and concepts developed by the distributed computing community and examine their potential relevance to the development of networked computers.
\end{abstract}

Biography: David Peleg received the B.A. degree in 1980 from the Technion, Israel, and the Ph.D. degree in 1985 from the Weizmann Institute, Israel, in computer science. He then spent a post-doctoral period at IBM and at Stanford University. In 1988 he joined the Department of Computer Science and Applied Mathematics at The Weizmann Institute of Science, where he holds the Norman D. Cohen Professorial Chair of Computer Sciences. His research interests include distributed network algorithms, fault-tolerant computing, communication network theory, approximation algorithms and graph theory, and he is the author of a book titled "Distributed Computing: A Locality-Sensitive Approach," as well as numerous papers in these areas. 